\title{
Das Matthäusevangelium im Wandel der Horizonte: vom „Hause Israels“ $(10,6)$ zu „allen Völkern“ $(28,19)$
}

Vereinfacht gesagt hat das Matthäusevangelium (MtEv) zwei Gesichter: Ein jüdisches und ein antijüdisches. Unmittelbar damit verbunden ist die Stellung des Gesetzes: Einerseits soll es nicht ,aufgehoben“, sondern bis in die kleinsten Gebote ,,erfüllt“ werden; und da gibt es, wie an vielen anderen Stellen, gerade rabbinisch anklingende Ausdrücke (Mt 5,18-19). Andererseits wird gesagt, dass ,die Gerechtigkeit von Pharisäern und Schriftgelehrten“ nicht genügt, und dass Gott sich ,ein anderes Volk“ wählen wird, das ,die Früchte des Gottesreichs bringt“" $(5,20 ; 21,43)$. Zwischen diesen zwei Gesichtern besteht eine Spannung, die das ganze Evangelium durchzieht.

Mit dieser ganzen Spannung ist Matthäus aber auffallenderweise das prominenteste Evangelium der frühen Heidenkirche geworden, ausgenommen vielleicht in Kleinasien. Im heidenchristlichen Zusammenhang musste die Gesetzesfrage dann mit gesteigerter Dringlichkeit gestellt werden.

Hiermit muss eine andere Spannung zusammenhängen, die im matthäischen Drama eine herausragende Rolle spielt, und die wir hier thematisieren wollen: das Verhältnis der Jesusgemeinde zu den Nichtjuden. In Mt 10,5 f klingt es schroff innerjüdisch, wenn Jesus seine Boten ausschickt: „Geht nicht auf einem Weg zu den Heiden, betretet keine Stadt der Samariter, geht vielmehr zu den verlorenen Schafen des Hauses Israels.“ Laut 15,24 habe Jesus auch von sich selber gesagt, er sei ,nur zu den verlorenen Schafen des Hauses Israels gesandt“. In 28,19, ganz am Ende, sagt der Auferstandene dann aber ganz anders: „Geht hin, macht alle Heiden (Völker) zu meinen Jüngern ...“

Gerade in einer Zeit, in der Judaistik und neutestamentliche Wissenschaft in einer erfreulichen Vertrautheit zusammenarbeiten, sind solche Spannungen unüberhörbar. ${ }^{1}$ Es genügt nicht mehr, sie im Rahmen des Evangeliums zu harmonisieren, d.h. faktisch zu verkennen. Es wäre besser, sie erst einmal $\mathrm{zu}$ akzeptieren. Sodann sollen wir als

1 Vgl. G. N. Stanton, A Gospel for a New People. Studies in Matthew, Edinburgh 1993, 146: „Matthew's vigorous anti-Jewish polemic is acutely embarrassing to most modern readers of this gospel.“ 
Wissenschaftler und Lehrer der Kirche versuchen, sie aus inhaltlichen oder historischen Gründen zu verstehen.

Wichtig ist $\mathrm{zu}$ allererst die allgemeine Einsicht, dass Evangelien Gemeinschaftstexte sind, Texte, die aus der Tradition einer Gemeinschaft heraus, für diese Gemeinschaft selber, durch ihre Mitglieder geschaffen und rezipiert wurden; Texte also, deren Aussagekraft es nicht nur im theologischen, sondern auch im sozialen Kontext zu befragen gilt. ${ }^{2}$ Bei einem solchen Zuhören besagen die angeführten Passagen vieles über die soziale Zugehörigkeit des MtEv und über die möglichen Spannungen und Entwicklungen in diesen Bezügen. Hierbei haben Daten halachischer Art, also die jüdische Gesetzestradition betreffend, wegen ihrer spezifischen sozialen Relevanz einen hohen Informationswert. ${ }^{3}$

\section{Deutungsversuche}

Zuerst ein Blick auf die Auslegungsgeschichte. Zwei einflussreiche patristische Beispiele, die in ihrer einheitlichen Interpretation lehrreich sind, gehen voran.

Konsequent allegorisiert Origenes, der große christliche Philo-Exeget, „die verlorenen Schafe des Hauses Israels“ aus Mt 15,24, ${ }^{4}$ wie er gleichzeitig den universellen Missionsbefehl 28,19 moralisiert. In seinem Matthäuskommentar $(11,17)$ erklärt er die „Schafe“ als gengj yucwh doratikwh apol woj, ,verlorenes Geschlecht klarsehender Seelen“. Im Hintergrund steht hier Philos Deutung des Namens „Israel“ als to. or ati kon genoj, „das sehende Geschlecht", 5 nur dass Origenes, anders als Philo, dabei die Juden praktisch ausschließt. Das deutet er anderweitig an: Der Ausdruck der ,verlorenen Schafe Israels“ sei „,nicht nach dem Fleische zu verstehen“, so „wie es die geistig armen Ebioniten tun“. Die judenchristliche Deutung

2 Vgl. U. Luz, Das Evangelium nach Matthäus, EKK 1, 4 Bde., Düsseldorf u. Neukirchen ${ }^{5}$ 2002, ${ }^{4} 2007$, 1997, 2002, I, 94; Stanton, Gospel, 45-53; P. J. Tomson, „If this be from Heaven ...". Jesus and the New Testament Authors in their Relationship to Judaism, The Biblical Seminar 76, Sheffield 2001, 122-125.

3 Vgl. P. J. Tomson, The Halakhic Evidence of Didache 8 and Matthew 6 and the Didache Community's Relationship to Judaism, in: H. van de Sandt (Hg.), Matthew and the Didache. Two Documents from the Same Jewish-Christian Milieu? Assen u. Minneapolis 2005, 131-141. Vgl. auch Zetterholm und Bockmuehl, s. u. Anm. 82.

4 Vgl. auch W. D. Davies / D. C. Allison, A Critical and Exegetical Commentary on the Gospel according to St. Matthew, ICC, 3 Bde., Edinburgh 1988-1997, I, 551: Or.princ. 4,3,8. [Vgl. a.a.O. 165 über Allegorisierungen von Mt 10,6 außer bei Tertullian und Chrysostomus.]

5 Migr. 18; Fug. 140. 
wird also abgewiesen. ${ }^{6}$ Ebenfalls im Matthäuskommentar (10.18) legt Origenes Mt 28,19 aufgrund des allgemeinen Spruches Mt 13,57 aus, der Prophet sei nur im eigenen Lande und Hause nicht anerkannt: „Deshalb haben die Apostel Israel verlassen, und haben den Befehl des Erlösers ausgeführt: ,Macht alle Völker zu Jüngern“." Jesus sei also nur zum „geistlichen Israel“ gesandt, und die ungläubigen Juden sind nicht in die hoffnungsvolle Zukunft des Evangeliums einbegriffen.

Ähnliches, aber in historisierter Form, erfahren wir beim späteren Nachfolger des Origenes, Euseb. In seiner Kirchengeschichte (3.5.2) bezieht er den Missionsbefehl an die „Völker“" auf die Zerstörung der jüdischen Hauptstadt als Strafe Gottes für die Verfolgung Christi und seiner Apostel. ${ }^{7}$ Radikal historisiert und von matthäischen Zitaten unterstützt erscheint dasselbe in der Demonstratio evangelica (9.11): Jesus sei als Prophet ,zu den verlorenen Schafen des Hauses Israels“ gekommen. Die hätten seine Gnadenbotschaft aber nicht angenommen, weshalb der Herr ihnen gesagt habe: „Das Reich wird ihnen genommen und einem Volk, das seine Früchte bringt, gegeben werden“ (Mt 21,43). Nachdem er dann seinen Jüngern gesagt habe: „Geht hin, macht alle Völker zu meinen Jüngern“, seien jetzt „wir, die Heiden“ es, die den prophetischen Auftrag erfüllten, während das jüdische Volk seine von Gott auferlegte Strafe trage. Dieser Textauswahl beleuchtet stringent die antijudische Linie im MtEv.

Ein wichtiges Detail: Bei beiden Kirchenvätern bedeutet eqnh nicht im alttestamentlichen Sinn „Völker“, sondern „Nichtjuden“, dem mittelhebräischen, individualisierten גויים entsprechend. Beide Theologen vertreten eine exklusiv-heidenchristliche Deutung der Schrift, die das Halten des jüdischen Gesetzes im Prinzip ausschließt. ${ }^{8}$ Judenchristliche Elemente wie der Schafespruch werden allegorisiert oder in die Vergangenheit hinein historisiert. Das MtEv wird konsequent antijüdisch gelesen.

6 Comm. in Matth. 11,17ff; Comm. in Ioan. 20,5,39: ankata, to. sumati kon e ixh\$cqai ; De princ. 4.3.8: ouk edl anbanonen tauka wij oi vptwcoi , th/ d anoi a| IEbi wnai bi )))edi wn gar oyptwcoj parV Ebrai oij anonazetai .

7 Dies betrifft eine der vor-nizänischen Zitationen mit dem Kurztext: poreugentej maqhteusate panta ta. exnh en tw/ anomati, nou Seit F. C. Conybeare, The Eusebian form of the Text Matth. 28,19, ZNW 2, 1901, 275-288 haben manche Exegeten gemeint, dies sei die ursprüngliche Version, die Euseb nach Nizäa 325 aufgegeben habe und die unter kaiserlichem Druck aus allen erhaltenen Handschriften verschwunden sei. Aufgrund der langen Zitate an anderen Stellen bei Euseb schlägt M.-J. Lagrange, Évangile selon St. Matthieu, EtB, Paris ${ }^{4} 1927$, 544 vor, Euseb habe hier gekürzt, um das Unterrichten im Namen Christi zu betonen. Luz, Evangelium IV (s. Anm. 2), 431 Anm. 15 folgert aus der Handschriftenlage, sie biete „methodisch keine Möglichkeit“, den Kurztext für ursprünglich zu halten. Dennoch bleibt Conybeares Erklärung zu bedenken.

8 Vgl. die Mühe, die sich Eus.d.e. 1-5 gibt zu erklären, weshalb die Kirche die Schrift geerbt hat und dennoch das Gesetz nicht hält. 
Moderne Exegeten zeigen mehr Distanz zu Matthäus und mehr Verständnis für die alttestamentlich-jüdische Perspektive, die in diesem Evangelium eine wichtige Rolle spielt. Daneben ist der seit dem 19. Jahrhundert verbreitete Weltmissionsgedanke hinzugekommen, in den die Mission an die Juden wie selbstverständlich einbegriffen wird. ${ }^{9}$

Père Lagrange nimmt in seinem kenntnisreichen Matthäuskommentar $(1927)^{10}$ Mt 10,5 wie 28,19 traditionsgemäß für authentisch-jesuanisch und folgert hinsichtlich des letztgenannten Textes: „On comprend très bien que la restriction aux brebis d'Israël (Mt. x,5 s.) ait été levée en ce moment.“ Ähnlich wie Origenes es versteht, habe der Auferstandene die jesuanische Beschränkung auf Israel aufgehoben, aber eine Mission an die Juden noch immer vorausgesetzt. Wie die ihm ganz vertrauten Kirchenväter versteht Lagrange das matthäische eqnh durchaus als „gentils“ d.h. Nichtjuden. Harmonisierend wird hier eine Evolution innerhalb der matthäischen Erzählung vorausgesetzt.

Dagegen betonte Georg Strecker (1962) ${ }^{11}$ einen scharfen Gegensatz zwischen der judenchristlichen und der exklusiv-heidenchristlichen Schicht und stellte fest, dass die judenchristliche Schicht des MtEv „eine ältere Entwicklungsstufe des Gemeindelebens" repräsentiere, während die letzte Redaktion des Textes einer heidenchristlichen Orientierung entspreche. In Passagen wie Mt 8,5-13 und 21,33-22,14 komme die „heilsgeschichtliche Ablösung des jüdischen Volkes“ zum Vorschein. Strecker stellt hier kritisch die antijüdische Linie im Evangelium heraus, die Euseb seinerzeit bevorzugte. Nach Strecker habe der Evangelist in 15,24 und 10,6 absichtlich, aufgrund „historischer Reflexion“ (nämlich über die von Strecker öfter vorausgesetzte „Ablösung Israels") die judenchristlicher Tradition entnommenen Worte von der Sendung ,an die verlorenen Schafe des Hauses Israels“ eingefügt.

David Flusser, der das MtEv nur gelegentlich aufgegriffen hat, behauptet in seinem Artikel über „Zwei Beispiele antijüdischer Redaktion bei Matthäus“ (1975), ${ }^{12}$ das MtEv sei deshalb außergewöhnlich, weil wir in ihm einerseits „,echte antijüdische Passagen“ finden, es aber andererseits ,alten,

9 Vgl. für die Entwicklungen im Rheinland P. G. Aring, Christliche Judenmission. Ihre Geschichte und Problematik dargestellt und untersucht am Beispiel des evangelischen Rheinlandes, FJCD 4, Neukirchen 1980.

10 Lagrange, Évangile (s. Anm. 7), 544.

11 G. Strecker, Der Weg der Gerechtigkeit. Untersuchung zur Theologie des Matthäus, FRLANT 82, Göttingen ${ }^{3} 1971$, v. a. 15-35, „Der Redaktor - ein Judenchrist?““.

12 In: D. Flusser, Entdeckungen im Neuen Testament, Bd. 1: Jesusworte und ihre Überlieferung, hg. v. M. Majer, Neukirchen 1985, 78-96. Die Paragraphen I-III erschienen ursprünglich als: Two Anti-Jewish Montages in Matthew, Immanuel 5, 1975, 37-45; mit einem Neudruck dieses Artikels erschien Paragraph IV als „Matthew’s ,Verus Israel“ “ in: ders., Judaism and the Origins of Christianity, Jerusalem 1988, 552-560, 561-574. 
ursprünglichen Stoff“ und sogar „einige Perikopen in ihrer ursprünglicheren, jüdischen Fassung" enthalte. Insbesondere am Beispiel der Geschichte des Hauptmanns von Kafarnaum (Lk 7,1-10; Mt 8,5-13) und des Gleichnisses der bösen Winzer (Mk 12,1-12; Lk 20,9-18; Mt 21,22-46) werde klar, wie der matthäische Redaktor seinen Stoff im antijüdischen Sinne redigiert habe. Den geschichtlichen Ort der matthäischen Gemeinde hat Flusser durch Vergleich mit 5Esr und Justin dem Märtyrer gesucht. ${ }^{13}$

Der Kommentar von W. D. Davies und Dale C. Allison (1988) nimmt wegen der ,jüdischen Färbung “ des traditionellen und redaktionellen Materials und wegen der vermuteten Verwendung des hebräischen AT an, der „Autor“ des MtEv sei Jude gewesen. Im Hinblick auf den universellen Missionsbefehl 28,19 sei es jedoch „,merkwürdig“ (odd), dass 5,47 und an anderen Stellen „herablassend“ über die eqnikoi, gesprochen werde. Zwischen 10,5 und 28,19 bestehe eine Verbindung im wiederholten por enesqe eine Spannung wird hier aber nicht registriert. 28,19 werde der universelle Missionsbefehl gegeben, weil „die Auferstehung das Ende der exklusiven Konzentration auf Israel markiere“. Die „Weltmission“ aber „schließe Israel ein“. ${ }^{14}$ Die heuristische Zielvorstellung scheint Harmonie zu sein.

In seinem Buch „A Gospel for a New People“ (1993) legt Graham Stanton die Auffassung dar, dass der Verband von matthäischen Gemeinden den Bruch mit dem Judentum vollzogen habe und sich als ein „neues Volk“ sah, in das bereits manche Heiden aufgenommen waren. Die scharfe antipharisäische und antijüdische Polemik, die das ganze Evangelium durchzieht, diene dem Zweck, diese traumatisierte Gemeinschaft abzugrenzen und vor Verfolgung von Seiten der jüdischen Behörden zu schützen. In 21,43 zeige sich so ,am deutlichsten, dass die matthäische Gemeinschaft sich als eine gesonderte und ganz verschiedene Größe gegenüber dem Judentum sah". Die Mission an Israel bleibe aber in Kraft, weil panta ta. eqnh,„Individuen innerhalb Israels einschließe“.15

13 U. z. in einer „heidnisch-zionistischen Sekte“, die die Beobachtung des jüdischen Gesetzes mit einer antijüdischen Ausrichtung kombiniert habe. Flusser las m. E. die verschiedenen Schichten des MtEv allzu buchstäblich als einheitlichen. Die Nähe seiner Interpretation zu der Streckers hat er nachträglich hinzugefügt, s. Anm. $11 \mathrm{im}$ ursprünglichen Artikel und Anm. $23 \mathrm{im}$ Nachtrag. Auch Stanton, Gospel (s. Anm. 1), 256-277 hat das Verhältnis zu 5Esr ausführlich untersucht.

14 Davies / Allison, Commentary I (s. Anm. 4), 33.58.559; II, 167; III, 368.

15 Stanton, Gospel (s. Anm. 1), 124.146-168. F. Wilk, Jesus und die Völker in der Sicht der Synoptiker, BZNW 109, Berlin 2002, 126-131 vergleicht Mt 10,5-8 und 28,18 ff mit der Schlussfolgerung (129): In 28,19, ,wird der in 10,5-8 erteilte Sendungsauftrag Jesu weder ausgeweitet noch aufgehoben, sondern ergänzt." 
Ulrich Luz nimmt in zwei Aufsätzen (1989, 1993) im MtEv einen Antijudaismus wahr, der einem Bruch mit dem rabbinischen Judentum entspreche. ${ }^{16}$ Diesem sei eine Stufe von enger Verwandtschaft mit der pharisäischen Bewegung vorangegangen. Die Mission ,an den verlorenen Schafen des Hauses Israel“", bei der die Jünger nicht ,,auf einem Weg der Nichtjuden" gehen sollen (10,5-6), sei von der Mission ,an allen Nichtjuden“ oder „Völkern“ abgelöst worden $(28,19)$. Doch wird das nicht am ganzen Evangelium, sondern nur an einzelnen Passagen durchgeführt, wie Luz im 4. Band seines Matthäuskommentars (2002) nochmals betont hat. ${ }^{17}$ Die neugefasste Einleitung zum Kommentar ( $\left.{ }^{5} 2002\right)$ nennt das MtEv ein judenchristliches Evangelium, das ,eine deutliche Distanz zum pharisäisch-protorabbinischen Judentum“ erkennen lasse. Ein richtiger Bruch zeige sich, nochmals, in der Wende zur Heidenmission: „Der Missionsbefehl des auferstandenen wird dem Gebot des irdischen Jesus antithetisch gegenübergestellt." ${ }^{18}$ Hinsichtlich der Stellung der matthäischen Gemeinde im Judentum schließt sich Luz der Auffassung Stantons an, sie habe den Bruch mit der Synagoge bereits hinter sich, aber anders als Strecker meinte, sei der Redaktor kein geschichtstheologisch reflektierender Heidenchrist gewesen.

Strecker, Flusser, Stanton und Luz deuten also die inneren Spannungen des MtEv im Rahmen der Zeitgeschichte; Lagrange und Davies / Allison harmonisieren sie. Luz kommt der Wirklichkeit des MtEv wohl am nächsten, weil er die offensichtlichen Widersprüche und verborgenen Aporien dieses durchlebten Textes in ihrer Unvermitteltheit stehen lässt. Ein Blick auf die jüdische Geschichte des 1. Jahrhunderts, insbesondere auf die Ereignisse am Ende des Jahrhunderts um die „,Trennung“ von Juden und Christen soll helfen, die Spannungen innerhalb des MtEv besser zu begreifen.

\section{Nichtjuden in der Jesustradition}

Zunächst soll die Haltung Jesu selbst bzw. der ihm zugeschriebenen Tradition zu Nichtjuden ins Auge gefasst werden.. Während diese

16 U. Luz, Das Matthäusevangelium und die Perspektive einer biblischen Theologie, in: D. R. Daniels u.a., „Gesetz“ als Thema Biblischer Theologie, JBTh 4, Neukirchen-Vluyn 1989, 233-248; ders., Antijudaismus im Matthäusevangelium als historisches und theologisches Problem, EvTh 53, 1993, 310-327 (2005 in englischer Übersetzung publiziert).

17 Auch die Bedeutung von eqnh - „Nichtjuden“/,Völker“ - ist nach Luz nicht eindeutig, vgl. Luz, Evangelium IV (s. Anm. 2), 449-454.

18 Luz, Evangelium I, 88.91. 
Tradition, die hypothetische erste Schicht des Evangeliums, meistens schwer zu beschreiben ist, lässt sich die Position Jesu jedoch in diesem Zusammenhang ziemlich leicht von ihrem Überlieferungszusammenhang unterscheiden. Mit dem Ergebnis werden wir dann nachher den Werdegang des MtEv besser beschreiben können.

Vorausgeschickt werden muss, dass es im Frühudentum unterschiedliche Haltungen gegenüber Nichtjuden gab. Die Qumranschriften machen den Eindruck, dass die mit ihnen zu assoziierenden Essener äußerst abweisend waren. Laut ihrer Halacha sind Heiden lauter Götzendiener, mit denen z. B. kein Handel getrieben werden darf. ${ }^{19}$ Sofern wir die Halacha der zeitgenössischen Pharisäer rekonstruieren können, war ihre Haltung insgesamt pragmatischer. ${ }^{20}$ Von den beiden Pharisäerschulen zeichnete sich aber die konservativere Schule Schammais durch eine größere Reserve gegenüber Nichtjuden aus. ${ }^{21}$

Auch müssen wir uns darüber verständigen, was wir unter „Jesustradition“ verstehen, und wie sie erschlossen werden soll. Wahrscheinlich hat die Tradition der Jesusworte, wie die anderer frühüdischer Lehrer, sich in mündlicher Form gebildet und fortgesetzt. ${ }^{22}$ Besonders an der rabbinischen Literatur lassen sich in einem solchen Prozess zwei entgegengesetzte Tendenzen wahrnehmen: ein Streben nach Bündigkeit und Uniformität, und eine bewusste oder unbewusste Neigung zur Anpassung an neue Umstände. Dabei lässt sich sehr wenig von individuellen Autoren sprechen. ${ }^{23}$ Der „Autor“ eines Spruchs hat wohl wichtige Elemente von seinen Lehrern übernommen; vielleicht gelingt es erst einem seiner Schüler, die vollendete Form zu finden. Dennoch nennt man es, nicht ganz zu Unrecht, ,seinen“ Spruch. Oft spricht man aber besser von „der Tradition des Rabbi NN“. Das gilt für viele Lehrer des 1. Jahrhunderts, deren Worte die rabbinische Literatur aufbewahrt. Wenn wir diese relative Unsicherheit akzeptieren, sind wir doch manchmal imstande, inmitten der so überlieferten Geschichten und Sprüche

19 CD 12,6-11 (Handel mit Nichtjuden); 4Q174 1 i 3-4 (Nichtjuden und Proselyten im Tempel).

20 Vgl. die rabbinische Halacha in Sachen Handel, mAS 1,5-8. Nach Flav.Jos.Bell. 2,409 war es normal, dass Opfer von Nichtjuden im Tempel angenommen wurden.

21 Vgl. mChul 2,7: R. Eliezer (bekannter Schammait) verbietet es, für einen Nichtjuden zu schlachten, denn „die normale Einstellung des Nichtjuden ist Götzendienst“. Weitere Belege s. u., Abschnitt 3.

22 Nach aller Polemik der letzten Jahrzehnte scheint es mir klar, dass das (mit einem Vorwort von J. Neusner!) neu herausgegebene Buch von B. Gerhardsson, Memory and Manuscript. Oral Tradition and Written Transmission in Rabbinic Judaism and Early Christianity, Uppsala 1961, Grand Rapids 1998, auf seine umfassende Würdigung und Auswertung noch wartet.

23 Vgl. für dieses und andere Kennzeichen S. Safrai, Oral Tora, in: ders. (Hg.), The Literature of the Sages, Bd. 1: Oral Tora, Halakha, Mishna, Tosefta, Talmud, External Tractates, CRI II.3a, Assen u. Philadelphia 1987, 35-119. 
charakteristische Züge einer Gestalt zu erkennen, wie z. B. der Hillels oder Jochanan ben Zakkais, ${ }^{24}$ wenn auch nicht ein genaues Bild dieser Person in all ihren Einzelkeiten.

Im Fall der Worte Jesu ist der Abstand zwischen „Urheber“ und Redaktor kürzer und die Möglichkeit der Vermischung mit den Worten anderer Lehrer geringer, aber auch hier gibt es einen Unsicherheitsfaktor, der es im allgemeinen ratsam macht, von der „Tradition Jesu“ zu sprechen. Doch geschieht es auch hier bei Gelegenheit, dass ein Wort dieses jüdischen Lehrers im Überlieferungskontext einen so außerordentlichen oder originellen Klang hat, dass sich der Eindruck von Authentizität aufdrängt. Das ist bei unserem Thema der Fall. Es gibt einige von Matthäus unabhängige synoptische Geschichten, die wertvolle Informationen über die Jesu zugeschriebene Haltung zu den Nichtjuden vermitteln.

Im wohl für Nichtjuden konzipierten Markusevangelium ${ }^{25}$ zeichnet sich die Geschichte 7,24-30 durch eine unerwartete Abneigung gegen die Heiden aus. Jesus wanderte ins Gebiet von Tyrus, wo er ,in einem Hause verblieb und niemanden sehen wollte", also anscheinend in einem jüdischen Hause; das finden wir sogar nur im MkEv, nicht im ,judenchristlichen“ MtEv. Dennoch weiß ihn „eine Griechin, eine Syrophönizierin“ zu finden, die ihn bittet, ihre Tochter von einem Dämon zu befreien. Jesu Antwort, es gezieme nicht, „das Brot der Kinder den Hunden zu geben“ (7,27), hat einen so heidenunfreundlichen Klang, dass sie unmöglich der nichtjüdischen Gemeindetradition entstammen kann. Die Erwiderung der Frau, dass die Hunde die unter dem Tische gefallenen Brotstücke fressen, überzeugt Jesus aber - und das überrascht wiederum die späteren Leser.

Ebenfalls im MkEv befindet sich die Geschichte des im Lande der Schweinehüter herumwandernden Besessenen (5,1-20). Obzwar weniger beachtet, ${ }^{26}$ begegnet uns in dieser offenbar nichtjüdischen Umgebung nochmals die bemerkenswerte Zurückhaltung Jesu. Als er nach dem Exorzismus wieder ins Boot stieg, bat ihn der Geheilte, bei ihm bleiben zu dürfen. „Aber Jesus erlaubte es ihm nicht, sondern sagte: Geh nach Hause, und berichte den Deinen alles, was der Herr für dich getan“ $(5,18 \mathrm{f})$. Einzigartig ist diese Weigerung Jesu, jemanden ihm folgen zu lassen. Sie

24 Vgl. wegweisend D. Flusser, Hillels Selbstverständnis und Jesus, in: ders., Entdeckungen I (s. Anm. 12), 210-215. Vgl. auch die verschiedenen Aufsätze in J. H. Charlesworth / L. L. Johns (Hg.), Hillel and Jesus. Comparative Studies of Two Major Religious Leaders, Minneapolis 1997.

25 Vgl. Irenäus bei Eus.h.e. 5,8,3 f: Markus habe die Predigt Petri, der auch in Rom gepredigt habe, schriftlich abgefasst. Eine Ausrichtung auf Nichtjuden geht eindeutig aus dem Text selber hervor, wie etwa in der Erklärung jüdischer Reinheitsbräuche Mk 7,3-4 und in der nichtjüdischen Fassung des Ehescheidungsverbots 10,12; vgl. 1Kor 7,10.

26 Kennzeichnend ist etwa die Erklärung von S. Légasse, L'Évangile de Marc, LeDiv Commentaires 5, Paris 1997, 329: „L'heure de la mission universelle n'a pas encore sonné“ (vgl. Lagrange zu Mt 28,19: oben S. 4). 
hängt anscheinend mit dessen Nichtjudesein zusammen. Jesus sendet ihn dagegen ,zu den Seinen“, um ihnen über die wunderbare Heilung zu erzählen. Die Heidenmission wird von einem geheilten Heiden begonnen. ${ }^{27}$ Das passt natürlich gut zu Markus, liegt aber auch auf der Hand.

In der lukanischen Überlieferung finden sich zwei Geschichten von einem nichtjüdischen Hauptmann, die als Beispiel der lukanischen Dubletten-Technik betrachtet werden können (Lk 7,1-10; Apg 10,1-11,18). ${ }^{28}$ An sich sollte das die Frage der Authentizität nicht präjudizieren. Der Autor arrangiert empfangene Traditionen ungezwungen $\mathrm{zu}$ einer einheitlichen Erzählung und erreicht so einen hohen Grad von verisimilitudo, „Wahrscheinlichkeit“. ${ }^{29}$ Höchstens könnte man ihm die Tendenz vorhalten, Konflikte zu „deeskalieren“, wie etwa den zwischen Paulus und Barnabas Apg 15,39f. Andererseits verwendet er offensichtlich bewusst „authentisches“ Vokabular der Zeitgeschichte. ${ }^{30}$ Dass die Beschreibung des guten Verhältnisses zwischen den zwei Hauptleuten und ihren jeweiligen jüdischen Stadtgenossen (Lk 7,3-5; Apg 10,2.22) überlieferter historischer Wirklichkeit entsprechen könnte, bestätigen archäologische Daten über die Koexistenz von Juden und „Gottesfürchtigen“. ${ }^{31}$

Zudem muss das Anliegen des Autors bedacht werden, die „harmonische“ Aufnahme der Nichtjuden in die Jesusgeschichte zu unterstreichen. Völlig klar wird das in der zweiten, paulinischen Hälfte der Apostelgeschichte, es wird aber schon in den Berichten über die Hauptleute angekündigt. Daneben steht aber die Zurückhaltung an der Grenze zwischen Juden und Nichtjuden, die Jesus wie seinen wichtigsten Jünger Petrus kennzeichnet. Jesus betritt das Haus des Nichtjuden nicht, spricht

27 Wilk, Jesus (s. Anm. 15), 66 sieht, wie viele andere, im ehemaligen Besessenen einen Juden und liest hier einen für Mk außergewöhnlichen Befehl zur Verkündigung an die Heiden.

28 Über Dubletten (,pairs“) vgl. H. J. Cadbury, The Making of Luke-Acts [1927], Neudr. London 1958, 231-234.

29 Wichtig ist z. B., dass das lukanische Bild der jüdischen „Sekten“ mit dem des Josephus und der rabbinischen Literatur übereinstimmt, vgl. S. Mason, Chief Priests, Sadducees, Pharisees and Sanhedrin in Acts, in: R. Bauckham (Hg.), The Book of Acts in its Palestinian Setting, Carlisle 1995, 115-177; P. J. Tomson, Les systèmes de halakha du Contre Apion et des Antiquités, in: F. Siegert / J. U. Kalms (Hg.), Internationales Josephus-Kolloquium Paris 2001. Studies on the Antiquities of Josephus; Études sur les Antiquités de Josèphe, MJSt 12, Münster 2002, 189-220.

30 Wie cristoj kuriou Lk 2,26 oder paij [qeoul] Apg 3,13.26; 4,27, vgl. die kritische Bemerkung über Cristi anoi,Apg 11,26; vgl. M. Hengel, Zur urchristlichen Geschichtsschreibung, Stuttgart 1979, 57; Cadbury, Making, 229 f; E. Plümacher, Lukas als hellenistischer Schriftsteller. Studien zur Apostelgeschichte, StUNT 9, Göttingen 1992, 72-79 betrachtet dies als das typisch hellenistische Phänomen der „Archaisierung“, rechnet aber damit, dass Lukas dafür in der Tat alte Überlieferungselemente verwendet.

31 Berühmt ist das Beispiel von Aphrodisias, vgl. J. Reynolds / R. Tannenbaum, Jews and Godfearers at Aphrodisias. Greek Inscriptions with Commentary, Cambridge 1987, mit der Liste von ,gottesfürchtigen“ Synagogenmitgliedern. 
sogar (ungleich der matthäischen Parallele) überhaupt nicht mit ihm, und Petrus braucht den dreimal wiederholten, schockierenden Traum, um über die Schwelle gezogen $\mathrm{zu}$ werden. Überraschenderweise scheint der Hauptmann aus Kafarnaum das zu verstehen und ihm zuvorzukommen: „Herr, ... ich bin nicht geeignet, dass du unter mein Dach kommst“ (Lk $7,6) .32$

Im Hintergrund steht anscheinend hier wie in Apg 10 das halachische Prinzip der טומאת נוכרים, ,Unreinheit der Nichtjuden“, insbesondere die spezielle Kategorie des מדור העמים, der ,heidnischen Wohnung“, die wie die Leichenunreinheit durch אהילות, „Überzeltung“, übertragen wird: Alles, was sich im „Zelt" befindet, wird unrein, und deshalb solle nach bestimmter Auffassung ein frommer Jude solch ein Haus nicht betreten. Der von Lukas (Q) verwendete Ausdruck ,unter mein Dach“ ist insofern sachgemäß, und ebenso das vom Hauptmann gebrauchte i kanoj, „geeignet“. ${ }^{33}$ Juden waren sich hierüber aber gar nicht einig; zudem ist eine allmähliche Verschiebung der Erklärungsgründe zu beobachten. ${ }^{34}$ Auch auf diesem Gebiet scheint übrigens die konservativere Schule Schammais zurückhaltender gegenüber Nichtjuden gewesen zu sein. ${ }^{35}$

Nach den lukanischen Berichten teilte Jesus eine ähnliche konservativere Auffassung, sowie, anfänglich, sein Jünger Petrus. Diese Auffassung steht der Tendenz des Autors, die Aufnahme von Nichtjuden in die Jesusbewegung zu begrüßen, entgegen. Nehmen wir die beiden Markusberichte dazu, dann stellt sich die relative Geschlossenheit der Jesustradition gegenüber Nichtjuden heraus. Sie ist anscheinend in der frühen „Apostelgeschichte“ beim Hinzukommen einzelner Nichtjuden im Schwanken geraten. Bei näherer Betrachtung ist die in der Apostelgeschichte implizierte Botschaft, dass Petrus für die Entwicklung der Heidenmission der Haltung seines Meisters untreu werden musste, ein fast unwidersprechliches Argument für die Authentizität dieser Jesustradition: Jesus hat sich ja gegenüber Nichtjuden reservierter verhalten als z. B. Philippus, der spätere Petrus und natürlich Paulus. Das steht

32 Ähnlich, auch zum Folgenden J. A. Fitzmyer, The Gospel According to St. Luke, 2 Bde., AncB 28, Garden City 1981-1985, 652.

33 Vgl. BDAG s. v. i kang: „,fit, appropriate, qualified ...“. Im Munde der Juden ist er aber ax oj $(7,4)$, obzwar er dem selber widerspricht: oude exauton hi wsa 7,7.

34 mOh 18,7-10; tAh 18,7-11. Alon machte plausibel, dass die Erklärung aufgrund von Totenunreinheit sekundär ist, weil nach Auffassung vieler eine nichtjüdische Leiche keine Unreinheit verursachte; die eigentliche Ursache sei vielmehr der vorausgesetzte Götzendienst: G. Alon, The Levitical Uncleanness of Gentiles, in: ders., Jews, Judaism and the Classical World. Studies in Jewish History in the Times of the Second Temple and the Talmud, Jerusalem 1977, 146-189, v. a. 183-186 und Anm. 75. Die Kritik J. Klawans' in seiner im Übrigen glänzenden Untersuchung Impurity and Sin in Ancient Judaism, Oxford 2000, $134 \mathrm{f}$ tut Alon m. E. Unrecht.

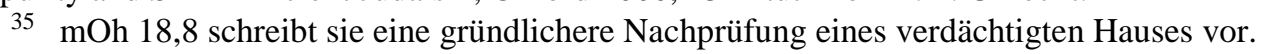


frühchristlichen Auffasssungen so sehr entgegen, dass nur eine grosse Autorität dahinter stehen kann: Jesus selbst.

Betrachten wir aufgrund obiger Schlussfolgerung die beiden matthäischen Sprüche über die Sendung zum „Hause Israels“, so können wir nicht ausschließen, dass sie ebenfalls aus der frühen Jesustradition stammen. Dies wird wahrscheinlich, wenn wir die Sendung Jesu vor ihrem alttestamentlich-jüdischen Hintergrund in den Blick nehmen.

Sowohl in Mt 10,6 als auch - in einem Zwischengespräch - in 15,24 wird den Jüngern gesagt, Jesus und seine Jünger seien ,vielmehr“ (nal/ I on, 10,6) oder „nur“ (ouk.. eivnh, 15,24) ejj ta. probata ta. apol w ota oi kou 'Israhl gesandt. Der Ausdruck probata apol w ota kommt aus Jeremia: Das Volk wird so bezeichnet, weil ,,ihre Hirten sie verführt haben“. ${ }^{36}$ Dem hat sich der semitisierende Ausdruck oikoj 'Israhl zugesellt. ${ }^{37}$ Semitisierend ist auch ouk.. e vnh, ${ }^{38}$ Der ganze Spruch Mt 15,24 muss aus einer bestimmten schriftlichen oder mündlichen Tradition stammen. ${ }^{39}$ Am ehesten doch wohl aus der Jesustradition; in welchem Maße Jesus selbst Vorgefundenes weitergebildet hat, kann dahingestellt bleiben. Der polemische Untergrund des Spruchs kann jedenfalls gut mit dem ,geheimen Programm“ zu tun haben, das auch aus anderen seiner Sprüche hervorgeht: seinem Streit mit der Verwaltung des Jerusalemer Tempels, auf den seine ganze Sendung hinauslief, ${ }^{40}$ der im prophetischen Akt der Tempelreinigung symbolisiert wurde. ${ }^{41}$ Das scheint ein seltsamer, bei $\mathrm{Lk}$ überlieferter Rätselspruch zusammenzufassen: „Es geht nicht an, dass ein Prophet außerhalb Jerusalems umkommt"“ $(13,33)$.

Nach der ihm zugehörigen Überlieferung meinte Jesus also im Anschluss an alttestamentlich-jüdische prophetische Traditionen, er sei nur gesandt,

\footnotetext{
36 Jer 27,6 (MT 50,6): probata apol w ota egenhoh o' I aoj noul à 'poi nenej autwh exwan autouj. Lk 15,6: to. probaton nou to. apol woj entstammt eher Ps 119,176: eql anhohn wi

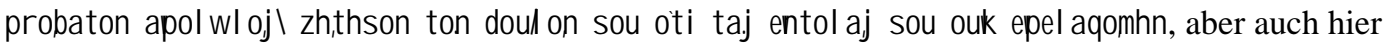
ist das Umherirren in Ermangelung guter Leitung impliziert.

37 Lagrange, Évangile (s. Anm. 7), 197: „oi kou sans article, comme un mot sémitique à l'état construit"; ähnlich BDR $\S 262,2$.

38 BDR § 376,1 - und zwar nicht nur ,in Anlehnung an das Aram.“, sondern ganz klar auch ans Hebr.: אלא/אין ... אל אל א. Nach M. H. Segal, A Grammar of Mishnaic Hebrew, Oxford 1927 (Neudr. 1970), 237 (§505f) sei אלא äquivalent mit bibl. אם - also genau év nh, Davies / Allison, Commentary II (s. Anm. 4), 550 nennen noch apostel I w ejj + die Betroffenen als Semitismus (nach Jeremias).

39 oi koj 'Israhl wird nur noch Apg 2,36 außerhalb biblischer Zitate verwendet (vgl. Apg 7,42; Hebr 8,8.10) und soll dort den Eindruck empfangener Tradition zu vermitteln.

40 Dem entspricht außerhalb der polemischen Rätselspruch Lk 13,31-33 auch der Davidssohnspruch Mk 12,35-37 (Lk 20,41-44), der in Apg 2,34-36 aufgenommen wird.

41 S. v. a. Mk 11,11-18 mit einzigartigen und aufschlussreichen Elementen. Die Weissagung der Tempelzerstörung gehört auch hierher, vgl. C. A. Evans, Predictions of the Destruction of the Herodian Temple in the Pseudepigrapha, Qumran, and Related Texts, JSP 10, 1992, 89-147.
} 
das ,in die Irre geführte Israel“ zu Umkehr und Heil aufzurufen. ${ }^{42}$ Im Hinblick auf die dargelegte polemische Ausrichtung hätten Heiden hier bloß eine negative Rolle: „Der Menschensohn wird den Hohenpriestern und Schriftgelehrten überliefert, und die werden ihn ... den Heiden überliefern " (Mk 10,33; Lk 18,32). Die oben zitierten mk und lk Entsprechungen des Schafespruchs machen es unwahrscheinlich, ihn als nur auf die Art der Mission Jesu bezogen zu verstehen. Es ist wahrscheinlicher, dass er auch mit seiner allgemeinen Reserve Nichtjuden gegenüber zusammenhängt.

\section{Die jüdische Geschichte im 1. Jahrhundert}

Um den zeitgeschichtlichen Rahmen des MtEv im Ganzen darzustellen, braucht es einen Überblick der jüdischen Geschichte im 1. Jahrhundert. Hierfür müssen wir uns auf die Untersuchungen sachkundiger Historiker stützen. Wir skizzieren insbesondere die zweite Hälfte des Jahrhunderts in drei aufeinander folgenden „Aufnahmen“.

(1) Hauptsächlich auf Josephus basiert die Information, die man in maßgebenden historischen Darstellungen des 1. Jahrhunderts findet, dass mit der Zeit der Prokuratoren $44 \mathrm{n}$. Chr. und v. a. nach 52 ein wachsendes Klima von Chaos und Gewalt entstand, aus dem schließlich der Krieg gegen Rom erwuchs. ${ }^{43}$ In der englischen Übersetzung des Schürer heißt es so: „Felix's term of office manifestly constitutes the turning-point in the drama which started in AD 44 and reached its bloody climax in AD 70.“44 Es liegt auf der Hand, hiermit die rabbinischen Berichte zu verbinden, die von der Vorkriegszeit als einer Periode „grundlosen Hasses“ sprechen..$^{45}$

42 Vgl. Lagrange, Évangile (s. Anm. 7), 197: „Le Messie était envoyé à Israël. Cette restriction est en harmonie avec xv,24, et très vraisemblable“ (d. h. keine Rejudaisierung). Insoweit hat auch Strecker, Weg (s. Anm. 11), 107-109 Recht, dieses vom Evangelisten „eingeschobene“ Wort einer Tradition ,judenchristlicher Theologie“ zuzuschreiben.

43 Vgl. E. Schürer, The History of the Jewish People in the Age of Jesus Christ, bearbeitet von G. Vermes u. a., Edinburgh 1973-1987, I, 455-470, mit generellem Verweis auf Ant. 22,1-16; 97-258; Bell. 2,204-308 und Tacitus ann. 15,44; hist. 5,9-10. Vgl. für diese Periode und weitere Literaturverweise (M. Stern, M. Hengel, E. M. Smallwood) auch P. J. Tomson, „Die Täter des Gesetzes werden gerechtfertigt werden“ (Röm 2,13) - Zu einer adäquaten Perspektive für den Römerbrief, in: M. Bachmann (Hg.), Lutherische und neue Paulusperspektive. Beiträge zu einem Schlüsselproblem der gegenwärtigen exegetischen Diskussion, WUNT 182, Tübingen 2005, 183221: 215-220. In seinem Nachwort im selben Band lehnt J. D. G. Dunn, The Dialogue Progresses, in: ebd., 388-430: 407 diesen Zeitrahmen mit einem simplen Verweis auf E. P. Sanders ab. Vgl. insbesondere die sicarii, ,Dolchmänner“, Bell. 2,247-279.

44 Schürer/Vermes I, 460.

45 שנאת חנם, tMen 13,22 = jJoma 1,1 [38c]; vgl. bJoma 9b; Kalla Rabbati 5,1. 
Es ist ebenfalls anzunehmen, dass sich die Vorherrschaft der Schule Schammais, von der die rabbinische Literatur berichtet und die, wie wir gleich sehen werden, beim Kriegsausbruch entscheidend war, in dieser Periode herausbildete. Die ihnen selbstverständliche Reserve gegenüber Nichtjuden hat anscheinend dazu beigetragen, dass die Schammaiten die wichtigsten Anstifter des Krieges wurden, dass sich also am meisten unter ihnen eine „zelotische“ Haltung abzeichnete - wie sie auch nach dem Kriege am stärksten dezimiert erscheinen. ${ }^{46}$

Sodann halte ich es für wahrscheinlich, dass diese wachsende heidenfeindliche Atmosphäre auch auf die Kirche eingewirkt hat und dass namentlich die Gesetzesfrage dadurch eine gesteigerte Dringlichkeit bekam. Die Paulusbriefe spiegeln einen wachsenden Druck auf die Nichtjuden wider, sich beschneiden zu lassen und das jüdische Gesetz auf sich zu nehmen. ${ }^{47}$ Diese Entwicklung kann man vermutlich an der Apg ablesen. ${ }^{48}$

(2) War unsere erste Aufnahme also eher eine „Zeitaufnahme“ der Jahre um 50, ist die zweite eine Momentaufnahme: der Kriegsausbruch 66 n. Chr. Nach der einleuchtenden Rekonstruktion von Heinrich Graetz ist der Tempelkommandant Eleazar, der Sohn des Hohenpriesters Ananias, der nach Josephus die Initiative zum Krieg ergriff, ${ }^{49}$ identisch mit Eleazar ben Chananja, dem führenden Nationalisten und Repräsentanten der Schule Schammais in der rabbinischen Literatur. ${ }^{50}$ In dieser Zeit muss auch die „Räubersynode“" (so Graetz ironisch, nach der gewaltsamen Synode von Ephesus 449) im Hause des Vaters von Eleazar, Ananias, stattgefunden haben, auf der nach rabbinischen Texten die Schammaiten den Hilleliten mit Waffengewalt eine Anzahl v. a. antiheidnischer Maßnahmen auferlegten. ${ }^{51} \mathrm{Gab}$ es somit einen Bürgerkrieg unter den Juden Palästinas, so

46 M. Hengel, Die Zeloten. Untersuchungen zur jüdischen Freiheitsbewegung in der Zeit von Herodes I bis 70 n. Chr., AGJU 1, Leiden ${ }^{2}$ 1976; I. Ben Shalom, The School of Shammai and the Zealots' Struggle against Rome, Jerusalem 1993 (hebr).

47 Vgl. den einschlägigen Unterschied zwischen 1 und 2Kor; ferner Gal, Phil, Kol und Röm.

48 Für weitere Hinweise vgl. Tomson, „Die Täter“ (s. Anm. 43), 215-220.

49 Bell. 2,409f; H. Graetz, Geschichte der Juden von den ältesten Zeiten bis auf die Gegenwart III.2, Leipzig 1888, 470-472, und Anm. 24, 26, 27 (795 ff).

50 Ihm werden MekhJ Yitro Ba-1/2odesh 7 [229 Horovitz / Rabin] die gleichen Sabbatsitten zugeschrieben wie Schammai MekhSh [148 Epstein / Melamed]; bBez 16a; PesR 23 [115b Friedmann]. Auch soll er die ursprünglich hasmonäische Daten festhaltende Fastenrolle „geschrieben“ haben, d. h. um zeitgenössische Ereignisse ergänzt: Scholion zu MegTaan, hg. v. H. Lichtenstein, in: HUCA 8-9 (1931-32) 257-351, hier 351; vgl. bShab 13b. Vgl. jetzt den hervorragenden Artikel von V. Noam, Megillat Taanit - the Scroll of Fasting, in: S. Safrai u.a. (Hg.), The Literature of the Sages II: Midrash and Targum; Liturgy, Poetry, Mysticism; Contracts, Inscriptions, Ancient Science; and the Languages of Rabbinic Literature, CRI II.3b, Assen u. Minneapolis 2006, 339-362, die aber Graetz' Rekonstruktion m. E. zu skeptisch beurteilt.

51 S. mShab 1,4; tShab 1,15 f; jShab 1,7 [3c-d]. Zu dieser Episode vgl. Graetz, Geschichte, ebd.; Hengel, Zeloten (s. Anm. 46), 204-211; A. Goldberg, Commentary to the Mishna Shabbat, 
kam es auch in der angrenzenden Diaspora $\mathrm{zu}$ blutigen Auseinandersetzungen zwischen Juden und Nichtjuden, z.B. in Antiochien. ${ }^{52}$ Wie es unlängst im ehemaligen Jugoslavien dramatisch zu beobachten war, führte auch damals der Kriegsausbruch $\mathrm{zu}$ einer mehrfachen sozialen Spaltung. Es ist wiederum anzunehmen, dass diese Spaltung nicht an der Kirche vorüberging und dass die bereits bestehenden Spannungen zwischen zelotischen und anderen Judenchristen sowie zwischen Juden- und Heidenchristen sehr stark anwuchsen.

(3) Die dritte Aufnahme machen wir in der Amtszeit Rabban Gamli'els des Jüngeren, der wohl der Enkel des Gamli'el aus der Apg war. Die hierfür verwertbaren rabbinischen Quellen müssen mit externen Quellen und archäologischen Daten kritisch verglichen werden; wir folgen hier insbesondere die - gewiss nicht unbestrittene - Synthese von Gedalyahu Alon. ${ }^{53}$ Die Amtsperiode Gamli'els hat wahrscheinlich erst nach dem Tode des Kaisers Domitian 96 n. Chr. angefangen, also um 100. In der rabbinischen Literatur wird er als ein herrschsüchtiger Charakter dargestellt, der seine Gegner hart anzupacken wusste. Zudem scheint er die Anerkennung der römischen Behörden erlangt zu haben, was seine Macht noch gesteigert haben muss. Gewissermaßen der richtige Mensch zur richtigen Zeit, hat er anscheinend die von seinem Vorgänger Jochanan ben Zakkai angefangenen Konsolidierungspolitik weitergeführt und die Gestaltung des jetzt entstehenden rabbinischen Judentums energisch angefasst.

Zu den Maßregeln, die Gamli'el II wohl zugeschrieben werden können, gehören der Pessachseder, d.h. die Hausliturgie des Passahmahles, das täglich dreimal $\mathrm{zu}$ betende Achtzehnbittengebet, und der Kanon der heiligen Schriften. ${ }^{54}$ Mit dem Achtzehnbittengebet verbunden war wahrscheinlich das „Trennungsdekret“, d.h. eine oder mehrere Maßnahmen, die die Trennung der Juden- und Heidenchristen von der

Jerusalem 1976 (hebr.); P. J. Tomson, Paul and the Jewish Law. Halakha in the Letters of the Apostle to the Gentiles, CRI III.1, Assen u. Minneapolis 1990, 173-177.

52 Flav.Jos.Bell. 7,45-53.

53 G. Alon, The Jews in Their Land in the Talmudic Age I, Jerusalem 1980, 107-124; zur Datierung vgl. S. Safrai, In Times of Temple and Mishnah. Studies in Jewish History, Jerusalem 1996 (hebr.), 331 f. Das Problem der Reisen der rabbinischen Führer nach Rom spielt hier eine wichtige Rolle; vgl. Alon, ebd.; Safrai, ebd., 365-381. Zur Diskussion über die „zionistische“ Anschauung Alons vgl. jetzt P. J. Tomson, Transformations of Post-70 Judaism. Scholarly Reconstructions and their Implications for our Perception of Matthew, Didache, and James, in: J. Zangenberg / H. van de Sandt (Hg.), Matthew, James and the Didache. Three Related JewishChristian Documents in their Historical, Social and Religious Setting (in Vorb.). W. D. Davies, The Setting of the Sermon on the Mount, Cambridge 1966, 256-315 vermittelt, wie Luz, Evangelium I (s. Anm. 2), 98 Anm. 261 bemerkt, eine „verdichtete“ Vorstellung der Periode von „Jamnia“, in der Frühes und Spätes unkritisch durcheinander verwendet wird.

54 Alon, ebd., 253-287. 
jüdischen Gemeinschaft zum Zweck hatten; die Einfügung der birkat haminim gehörte wohl dazu. ${ }^{55}$ Ungefähr in dieselbe Zeit muss auch die Einführung des Titels „Rabbi“ für ordinierte Toralehrer datiert werden; Lehrer, die vor der Tempelzerstörung wirkten, tragen ja keinen solchen Titel, wohl aber die späteren, ${ }^{56}$ und das stimmt gerade mit der noch zu nennenden Passus Mt 23,8 überein. ${ }^{57}$ Zusammen mit den anderen Maßnahmen markiert sie die Entstehung des „rabbinischen“ Judentums. In diesen Jahren oder gar bereits unter Jochanan ben Zakkai scheint der mündliche Redaktionsprozess der rabbinischen Tradition begonnen zu haben, der mehr als ein Jahrhundert später in die Fertigstellung der Mischna münden sollte, der Kernschrift des rabbinischen Judentums.

Ein Rückblick auf das Jahrhundert zeigt, wie aus dem Chaos der Vorkriegs- und Kriegsjahre die mehr oder weniger festumrissene Gestalt des rabbinischen Judentums hervortritt. Von Uniformität zu reden wäre aber unangebracht; schon die unzähligen Meinungsverschiedenheiten in der Mischna vermitteln den Eindruck großer Vielfalt. Nichtsdestoweniger stehen wir vor einem ungeheuren Sichtungsprozess, in dem die Kirche aus Juden und Nichtjuden wohl überaus schwerem Druck ausgesetzt war. In diesem Schmelztiegel hat offenbar das MtEv seine Form finden müssen.

\section{Die Entstehung des MtEv}

Es liegt auf der Hand, die großen Spannungen, die das MtEv in der Frage des Gesetzes und des Verhältnisses zu den Nichtjuden erkennen lässt, im Rahmen dieses gerade in dieser Frage so bewegten Jahrhunderts zu sehen. Es drängt sich die Vorstellung einer Entwicklung auf, in der die dramatische Abfolge von ganz verschiedenen Situationen $\mathrm{zu}$ einem schnellen Wandel von Anschauungen geführt hat. Die Eignung eines solchen evolutionären Erklärungsmodells steht, anders als im 19. Jahrhundert, nicht mehr im Voraus fest. Wenn es uns aber ermöglicht, die verschiedenen, z. T. widersprüchlichen Daten unseres Textes in einem einleuchtenden Zusammenhang zu sehen, kann es nicht übergangen werden. Zudem scheint die Differenz zwischen der Haltung der frühen

55 Weiteres s. in P. J. Tomson, The Wars against Rome, the Rise of Rabbinic Judaism and of Apostolic Gentile Christianity, and the Judaeo-Christians. Elements for a Synthesis, in: ders. / D. Lambers-Petry (Hg.), The Image of the Judaeo-Christians in Early Jewish and Christian Christian Literature, WUNT 158, Tübingen 2003, 1-31: 14-18.

56 Ähnlich Davies / Allison, Commentary I (s. Anm. 4), 135.

57 Der einzige Anhaltspunkt ist Mt 23,7-12, s. u. 
Jesustradition gegenüber Nichtjuden und der der späteren matthäischen Gemeinde so ein Modell anzuempfehlen.

Etwas schematisch stellen wir uns jetzt den Werdegang des Evangeliums in vier aufeinanderfolgenden Schichten vor, die sukzessiven Stadien in der Sozialgeschichte des MtEv entsprechen könnten. Wie gesagt haben sozialrelevante halachische Daten großen historischen Wert. Schließlich ergibt sich eine mögliche örtliche Bestimmung.

(a) Eine jesuanische Schicht. Texte aus dem MkEv und Lk-Apg zeigen die frühe Jesustradition eher zurückhaltend gegenüber den Nichtjuden. Daher könnte der nur im MtEv enthaltene Schafespruch auch dieser Tradition entstammen. Im Hinblick auf den alttestamentlichen Hintergrund des Spruches entspricht der Reserve gegenüber Nichtjuden positiv die Überzeugung, „der Menschensohn“ sei gesandt um das irregeführte Israel zurechtzubringen und seine falschen Führer in Jerusalem anzuklagen.

(b) Eine judenchristlich-pharisäische Schicht. Eine Nähe zur pharisäischen Tradition tut sich auf in matthäischen Ausdrücken wie „das Königreich der Himmel“ $(32 \times)$, ,die Zehnten von Minze, Dill und Kümmel“ (Mt 23,23), ,ein Schriftgelehrter, der ein Jünger des Reichs der Himmel geworden ist“ (13,52), „ein Iota oder Häkchen des Gesetzes“ $(5,18)$, die alle große Ähnlichkeit mit rabbinischem Traditionsgut aufweisen. Die schwer zu entscheidende Frage ist: Entstammen diese Ausdrücke bereits der matthäischen Redaktion oder noch der Jesustradition? Können die aufeinanderfolgenden matthäischen Redaktore nicht all diese Ausdrücke beibehalten haben, weil sie auch ihnen entgegenkamen, während der lukanische Redaktor sie für seine griechischen Leser meistens nicht brauchen konnte? ? $^{58}$

Entscheiden können wir erst, wo sich Unterschiede abzeichnen. Einigermaßen deutlich scheint das beim Vaterunser sein. Die bündige lukanische Version (Lk 12,1-4), die Jesus laut der narrativen Einleitung auf die Frage eines Jüngers lehrte, klingt mit ihrem charakteristischen Anfang „Vater" sicher ursprünglicher. Die matthäische Version dagegen hat nicht nur die Züge eines Gemeindegebets (,unser Vater ..."), sondern zudem matthäischen ${ }^{59}$ und „rabbinischen “60 Charakter (,,... der Du im Himmel“, Mt 6,9). Doch lässt sich nicht ausschließen, dass die betreffenden Redaktore eine Version des Gebets verwendet hat, die nicht nur in der Gemeinde

58 Das war auch die Arbeitshypothese David Flussers, die aber die Gegenhypothese einer matthäischen Redaktion nicht genügend einbezogen hat. - Im Fall des MtEv rechne ich mit mehreren, sukzessiven Redaktoren, während das vorliegende LkEv offensichtlich einen Endredaktor hatte.

59 Vgl. nuanciert Luz, Evangelium I (s. Anm. 2), 435 f

60 אבינו שבשמים ist rabbinisch, vgl. mSota 9,15 (3×) und zumal Seder Eliyahu (11 x); sehr häufig ist אביהםון שבשמים. 
verwurzelt war, sondern auch aus der Jesustradition stammte. ${ }^{61}$ Jedenfalls hat er sie mit dem wohl in der Tradition vorgefundenen Ausdruck zusammengestellt, der Jesus - wie wir jetzt besser wissen - vertraut gewesen sein kann: Dass seine Jünger beim Beten nicht „plappern“ sollen „wie die Heiden“ (Mt 6,7). ${ }^{62}$

Ganz klar ist der Unterschied zwischen Jesustradition und Matthäusredaktion beim Gebot der Ehescheidung, ${ }^{63}$ und zwar mit bezeichnender Wiederholung: Mt 5,32; 19,9.64 Es betrifft hier ein sozial höchst relevantes halachisches Element, weil die in der einschlägigen Literatur genannten „Unzuchtsklauseln“ genau mit der schammaitischen Halacha übereinstimmen (mGit 9,10), ${ }^{65}$ einerseits im Gegensatz zur hillelitischen, andererseits zur essenischen Auffassung. ${ }^{66}$ Strecker $^{67}$ hat hier richtig gesehen: Der matthäische Redaktor hat die schammaitischen Unzuchtsklauseln in die synoptisch-markinische Tradition eingeschoben. Man könnte hier von einer auffallenden „Schammaisierung“ sprechen. Anscheinend wollte der betreffende Redaktor die Ehescheidungslehre Jesu nicht als hillelitisch und auch nicht (wie bei Markus, Lukas und Paulus) als „essenisch“, sondern gerade als schammaitisch darstellen. Das bleibt erstaunlich, und man fragt sich, warum dem so ist.

(c) Eine antipharisäische Schicht. Der judenchristlich-pharisäischen Phase scheint eine antipharisäische gefolgt zu sein. Das erweist sich im deutlichen Kontrast zur oben herausgestellten Nähe zur pharisäischen Tradition. Dreimal im Abschnitt der Bergpredigt über Almosengeben, Beten und Fasten und sechsmal in der Rede gegen die „Schriftgelehrten und Pharisäer" wird durch Verwendung der Bezeichnung upokritai ,eine scharfe

61 Vgl. P. J. Tomson, The Lord's Prayer as a „Short Prayer“ from Pre-Rabbinic Judaism (in Vorb.).

62 Mt 6,7. Der Ausdruck kommt auch 5,47; 18,17 vor. Auf Zugehörigkeit zur Jesustradition weist der Kontrast zum dreifach umschließenden, sicher redaktionellen nh. wj oi ù̀okritai, 6,2.5.16, das Did 8,1.2 in genau entsprechender Funktion vorkommt.

63 Vgl. die ausführliche Behandlung in P. J. Tomson, Halakhah and the New Testament. A Research Overview Focussing on Divorce Law, in: R. Bieringer, F. Garcia Martinez, D. Pollefeyt, P.J. Tomson (Hg.), The New Testament and Rabbinic Literature, Supplements to the Journal for the Study of Judaism, Brill, Leiden, (in Vorb.). Vgl. auch die wichtigen traditionskritischen Bemerkungen von B. Schaller, Die Sprüche über Ehescheidung und Wiederheirat in der synoptischen Überlieferung [1970], in: ders., Fundamenta Judaica. Studien zum antiken Judentum und zum Neuen Testament, hg. v. L. Doering / A. Steudel, StUNT 25, Göttingen 2001, 104-124.

64 Vgl. die Liste von Wiederholungen, insbesondere Dubletten, in Davies/ Allison, Commentary I (s. Anm. 4), 89-91; Luz, Evangelium I (s. Anm. 2), 29 f.

65 Vgl. z. B. E. Neudecker, Das „Ehescheidungsgesetz“ von Dtn 24,1-4 nach altjüdischer Auslegung. Ein Beitrag zum Verständnis der neutestamentlichen Aussagen zur Ehescheidung, Bib. 75, 1994, 350-383.

66 J. A. Fitzmyer, The Matthean Divorce Texts and Some New Palestinian Evidence [1976], in: ders., To Advance the Gospel. New Testament Studies, New York 1981, 79-111.

67 Strecker, Weg (s. Anm. 11), 16 f. 
Abgrenzung gegenüber den Pharisäern vollzogen. Dies betrifft Lehren Jesu über religiöse Bräuche, die inhaltlich viel mit der pharisäischen Tradition gemein haben. ${ }^{68}$ Die Parallelen zwischen Mt 6,1-18 und Did 8 machen eine relative Datierung bezüglich der rabbinischen Tradition möglich, und zwar im Hinblick auf halachische Elemente, ${ }^{69}$ die auf die eingreifenden Maßnahmen Rabban Gamli'els Ende des 1. Jh. zu verweisen scheinen. ${ }^{70}$

Eine weitere Abgrenzung liegt im Jesus zugeschriebenen Verbot, sich offenbar amtlich - ràbdi, nennen zu lassen (Mt 23,8). Es wurde oben die Annahme ausgesprochen, dass dies ebenfalls eine Erneuerung aus der Zeit Gamli'els des Jüngeren (vielleicht auf seine Anregung) betrifft. ${ }^{71}$ Der matthäisch-redaktionelle Ursprung von 23,7-12 erscheint in den Ausdrücken o’ pathr o` ouvani oj (V. 9) und, erstaunlich, o`Cristoj (V. 10). Vielleicht sollte also richtiger von einer antirabbinischen Schicht des MtEv gesprochen werden. ${ }^{72}$ Auf das im JohEv erwähnte Trennungsdekret (Joh 9,22; 12,42; 18,3) gibt die Endform des MtEv jedoch keine Hinweise.

(d) Eine heidenchristlich-antijüdische Schicht. Schließlich hat sich im $\mathrm{MtEv}$ und also in der matthäischen Gemeinde ein heidenchristlichantijüdisches Denken niedergeschlagen. Im Prinzip ist das zu unterscheiden von der Öffnung zu den Nichtjuden, die an einigen Stellen zum Ausdruck kommt (Mt 10,18; 24,14; vielleicht auch 28,19?). Wie Paulus in seinen Briefen (Röm 9-11!) zeigt, braucht Heidenmission nicht notwendigerweise mit Antijudaismus einherzugehen.

In einigen matthäischen Passagen tut sich dann aber doch ein solcher auf. Zunächst erklingt in der Geschichte vom Hauptmann von Kafarnaum (Mt 8,5-13), in der die ganze Sympathie zwischen dem Hauptmann und den Juden aus der lukanischen Parallele fehlt, der verstörende Satz aus Jesu Mund, dass „die Kinder des Königreichs hinausgeworfen werden“, während vom Osten und Westen andere, anscheinend Nichtjuden, hinzutreten - was

68 Mt 6,2.5.16 (vgl. Did 8,1.2) - gemeint sind Pharisäer; 23,1.13.15.23.25.27.29 (vgl. 15,7; 22,18) sind Pharisäer direkt als „Heuchler“ angesprochen.

69 Dazu und zur Sache Tomson, Halakhic Evidence (s. Anm. 3).

70 Vgl. genauer Tomson, ebd. D. Flusser, Paul's Jewish-Christian Opponents in the Didache, in: S. Shaked u.a. (Hg.), Gilgul. Essays on Transformation, Revolution and Permanence in the History of Religions, FS Z. Werblowski, Leiden 1987, 71-90 sieht hier zu Unrecht eine heidenchristliche Abspaltung; ebenso, etwas abgeschwächt, H. van de Sandt/D. Flusser, The Didache. Its Jewish Sources and Its Place in Early Judaism and Christianity, CRI III.5, Assen u. Minneapolis 2002, 295 f. Fasten am Mittwoch und Freitag entspricht eher dem essenischen Solarkalender; das Festhalten am Vaterunser dem Widerstand gegen das jüngere Achtzehnbittengebet. Vgl. knapp Tomson, „If this be from Heaven ...“(Anm. 2), 388.

71 Vgl. Strecker, Weg (s. Anm. 11), 33, der hier aber ahistorisch eine „Ablösung vom Judentum“ sieht. Vgl. des Weiteren Tomson, The Wars against Rome (s. Anm. 55), 12 f u. Anm. 63.

72 Die Formulierung von Davies/Allison, Commentary I (s. Anm. 4), 137 trifft zu: „Matthew's gospel fits perfectly into the Jamnian period of Jewish reconsolidation.“ 
seine Überraschung über den Glauben des Hauptmanns ernsthaft beeinträchtigt. ${ }^{73}$ Sodann kommen im Gleichnis von den bösen Winzern nicht, wie in Mk 12,12 und Lk 20,19, die „Schriftgelehrten und Hohenpriester" in den Blick, sondern „die Hohepriester und Pharisäer“ (Mt 21,46), denen gesagt wird, dass „das Königreich einem anderen Volk gegeben wird, das seine Früchte bringt" $(21,43) .{ }^{74}$ Aus der von Verleumdung durchzogenen Geschichte von den Wächtern am Grab spricht sogar ein antijüdisches Ressentiment (Mt 27,62-66; 28,11-15), wenn es auch ein antichristliches Gerücht „unter Juden“ (para. loudai oij 28,15) herausstellen soll. ${ }^{75}$ Hier finden sich ebenfalls "die Hohepriester und Pharisäer" unhistorisch zusammen, sogar in der Gesellschaft des Pilatus $(27,62)$. Luz folgert aus Mt 21,43, der matthäische Redaktor sei „tatsächlich einer der Väter der kirchlichen ,Sukzessionstheorie“"; 28,15 sei solches aber nicht zu entnehmen. ${ }^{76}$ Diese Nuance erscheint mir wichtig zu sein. Die Wächtergeschichte setzt gerade in der zentralen Auferstehungsgeschichte einen ganz verfremdenden Akzent, entspricht aber eher ungebildetem populärem Empfinden. Weitere Retuschen in dieser Schicht sind quantitativ gering. Sie wurden anscheinend in einer letzten Redaktionsphase unsystematisch hinzugefügt und bezeugen, mit welchem Interesse das Evangelium damals gelesen wurde. Die früheren Schichten blieben dabei in aller Widersprüchlichkeit bestehen.

Für die Datierung des MtEv ergibt sich ein terminus ad quem aus Hinweisen auf Rezeption bei Ignatius, also bis ca. $110 \mathrm{n}$. Chr. ${ }^{77}$ Es gibt keine Spuren einer ähnlichen Rezeption in der mit dem MtEv eng verwandten Didache ${ }^{78}$ das $\mathrm{MtEv}$ ist also nicht früher als sie anzusetzen. ${ }^{79}$ Terminus a quo ist der beiden Texten gemeinsame Protest gegen das Gebet der „Heuchler“, das mit der Amtszeit Gamli’els II zu assoziieren ist. Es

\footnotetext{
73 Im Vergleich ist die lukanische Version kohärent: Jesus stand dem Nichtjuden reservierter gegenüber als die Kafarnaumer Juden!

74 Flusser, Beispiele (s. Anm. 12); Strecker, Weg (s. Anm. 11), 99-101, 110-113. Luz, Evangelium IV (s. Anm. 2), 216-229, mit Rückverweis auf Mt 8. Stanton, Gospel (s. Anm. 1), 151 meint zu Recht, dass die Verwerfung der Hohenpriester und Pharisäer mit ihrem Volk von den Exegeten oft übersehen wird.

75 Luz, Evangelium IV, 389-391， 420-427. Der Zusammenhang mit dem ebenfalls antijüdischen Petrusevangelium (vgl. $8-10)$ ist wichtig.

76 Luz, Evangelium III, 228; IV, 425.

77 Insbesondere IgnSm 1,1: bebapti snenon.. i h̀̀ pl hrwohy pała di kai osunh; vgl. Mt 3,15, aber auch die möglichen Anspielungen auf Röm 1,3 f; Lk 23,7 ff; ferner IgnPol 2,2 / Mt 10,16; IgnEph 19,2 f / Mt 2,2; vgl. R. E. Brown / J. P. Meier, Antioch and Rome. New Testament Cradles of Catholic Christianity, New York 1983, $24 \mathrm{f}$.

78 Tomson, Halakhic Evidence (s. Anm. 3); anders Van de Sandt / Flusser, oben Anm. 70.

79 Vgl. aber Luz, Evangelium I (Anm. 2), 103: Die Didache (v. a. Kap. 8) sei „fast sicher“ in einer „durch Mt geprägten Gemeinde entstanden“ und setze die matthäische Redaktion „Zweifellos“"voraus.
} 
ergibt sich eine Endredaktion um 100 n. Chr., also relativ spät. ${ }^{80}$ Das lässt aber genug Zeit für die Entstehung der Wächtergeschichte und der damit verwandt erscheinenden Pilatussaga 27,19.24f, die man als Anfang der langen Entwicklung volkstümlicher Evangelienapokryphen ansehen kann. ${ }^{81}$

\section{Gesamtschau}

Aus Obenstehendem geht hervor, dass die Entstehung der heidenchristlichantijüdischen Schicht mit der der antirabbinischen fast zusammengefallen ist. Wir müssen uns eine Kirchengemeinschaft vorstellen, in der eine beträchtliche Gruppe von Judenchristen noch in den Konflikt mit den eben hervorgetretenen „rabbinischen“ Behörden verwickelt war, als in einer anderen, heidenchristlichen Gruppe bereits antijüdische Geschichten umliefen. Unter den Heidenchristen existierte wohl schon die Vorstellung, dass Israel durch die (Heiden-)Kirche ersetzt wird. Genügend Stoff für Konflikte zwischen beiden Gruppen wäre dann vorhanden.

Dieses Profil passt gut zu John P. Meiers Beschreibung des syrischen Antiochien. ${ }^{82}$ In der bedeutenden antiochenischen Kirche sind die frühesten Spannungen zwischen Juden- und Heidenchristen bezeugt (Gal 2,11-14; Apg 15,1-5); eine christlich-zelotische, antipaulinische „Partei“ ist in den Quellen bezeugt. Nach dem Kriegsausbruch AD 66 brachen in dieser Stadt blutige Streitigkeiten zwischen Juden und Nichtjuden aus. ${ }^{83}$ Nach dem Jahrhundertwechsel trat der örtliche Bischof Ignatius, der früheste sichere Zeuge für das MtEv, im Konflikt zwischen Juden- und Heidenchristen entschieden auf die heidenchristliche Seite, obwohl er noch um das

80 Im Hinblick auf die heidenchristliche Endredaktion kommt Strecker, Weg (s. Anm. 11), 15-85 auf 95 n. Chr.; vgl. Stanton, Gospel (s. Anm. 1), 113-168 (Mitte der 80er Jahre); Luz, Evangelium I, $103 \mathrm{f}$ (,nicht lange nach 80“); Davies/ Allison, Commentary I (s. Anm. 4), 127 f.138 (70-100 bzw. 80-95).

81 Vgl. Luz, Evangelium IV, 268. Zur Wächtergeschichte vgl. das EvPetr, s. o. Anm. 75.

82 J. P. Meier, Antioch, in: Brown / Meier, Antioch and Rome, 11-86. Vgl. M. Zetterholm, The Formation of Christianity in Antioch. A Social-Scientific Approach to the Separation Between Judaism and Christianity, London 2003. Die soziale Verortung des MtEv S. 215 f würde m. E. natürlich anders ausfallen: Die Endfassung des MtEV entstammte dem ignazianischen Lager, die Didache dem von mir angenommenen ,lukanischen“: Tomson, „If this be from Heaven ..." (Anm. 2), 247-254. Für die politischen und halachischen Daten der früheren Periode grundlegend ist M. Bockmuehl, James, Israel and Antioch, in: ders., Jewish Law in Gentile Churches. Halakhah and the Beginning of Christian Public Ethics, Edinburgh 2000, 49-83; zuvor als: Antioch and James the Just, in: B. Chilton / C. A. Evans (Hg.), James the Just and Christian Origins, NT.S 98, Leiden 1999, 155-198.

83 S. o. Anm. 52. 
paulinische Ideal der einen Kirche aus Juden und Heiden wusste. ${ }^{84}$ Seit längerem Zeit hat man noch weitere Gründe für einen antiochenischen Ursprung des MtEv angeführt. ${ }^{85}$ Auch die römische Soldatensprache der Wächtergeschichte würde gut in die syrische Provinzhauptstadt passen, ${ }^{86}$ sowie, im Zusammenhang damit, die schroffe Antijudaisierung der Kafarnaumer Hauptmannsgeschichte.

So steht uns das MtEv im spannungsvollsten Sinne als „Gemeinschaftstext" vor Augen: als der Text einer Gemeinschaft, die, wohl in Antiochien, in kurzer Zeit dramatische Wandlungen des sozialen Horizontes erlebt hat. Fast gleichzeitig erklingen die heidenchristlichantijüdischen Verleumdungen und die judenchristlich-antirabbinischen Protestschreie; dahinter können wir die Stimmen der zelotischantipaulinischen Judenchristen hören und, bei genauem Zuhören, den leisen Nachklang der Jesustradition.

Nach der frühesten Überlieferung wusste Jesus sich mit seiner Verkündigung positiv zum ,,irregeführten Israel“" gesandt; dem entspricht negativ seine Zurückhaltung gegenüber Nichtjuden. Er musste erst von der phönizischen Mutter und vom römischen Hauptmann überzeugt werden, dass die Predigt des Gottesreichs auch für Heiden gilt. Seinen Jüngern lag dies schon näher; sie fanden neue Argumente, als Nichtjuden sich der Bewegung anzuschließen begannen. Eines des schönsten und wirksamsten war das vom pharisäisch geschulten Zeltmacher verwendete (stoisierende) Bild vom „einen Leib“ des gestorbenen und auferstandenen Messias aus jüdischen und nichtjüdischen Gliedern (1. Kor 12,12 f).

Die Zeitgeschichte kam dazwischen. „Eiferer für das Gesetz“ fingen an, die inklusiv denkenden Juden zu überschreien. In Judäa und Galiläa wuchs das Chaos, befördert durch die schwache römische Verwaltung. Es kam zur blutigen Auseinandersetzung, zum Krieg. Die Gesellschaft spaltete sich auf, Juden- und Heidenkirche fingen an, sich voneinander abzusetzen, die Rabbinen traten hervor und schufen Ordnung und Regel im eigenen Haus, und schließlich trat eine selbstbewusste, antijüdische Heidenkirche auf den Plan. Die Konstellation zweier sich gegenseitig abgrenzender und doch heimlich so verwandter Religionsgemeinschaften, Judentum und Christentum, bildete sich heraus, um sich jahrhundertelang zu behaupten.

Wieder ist aber die Zeitgeschichte dazwischengekommen. Während sie sich von den Erschütterungen eines massenmörderischen und neopaganen

84 IgnMagn 10,3; vgl. 8,1; 9,1; IgnPhil 6,1; die Kirche aus Juden und Heiden: IgnSm 1,2.

85 Zuerst behauptet von B. H. Streeter, vgl. Meier, Antioch, 22-27; Luz, Evangelium I (s. Anm. 2), 100-103 (faktisch eine Entscheidung für Antiochien); Davies / Allison, Commentary I (s. Anm. 4), 143-147.

86 koustwda $(27,65 \mathrm{f} ; 28,11)$, ein fast einzigartiger Latinismus, vgl. LSJ, s. v.; auch sunboul i on I anbane $n$ ist ein reiner Latinismus, vgl. BDR $\S 5,3 \mathrm{~b}$. 
22 Judaistik und neutestamentliche Wissenschaft - FS B. Schaller

Jahrhunderts am erholen ist, hat die heutige Heidenkirche vorsichtig angefangen, trotz überliefertem Antijudaismus und Antirabbinismus die Weisheit der pharisäischen Lehrer in einer neuen Perspektive zu sehen. So kann sie auch hoffen, die Botschaft immer besser zu begreifen, die auf Anstoß des galiläischen Lehrers ,,an die verlorenen Schafe des Hauses Israels“" ergeht sowie, genauso biblisch gesagt, ,,an alle Völker" ${ }^{67}$

87 Gerne gestehe ich meinen Dank an die Herausgeber des Bandes für ihre sorgfältige sprachliche und stilistische Verbesserung meines Artikels. 